\title{
Kiswahili usage in ICT in NEPAD secondary schools in Kenya
}

\author{
Jagero Juliet Akinyi
}

\begin{abstract}
There has been tremendous increase in Kiswahili usage in Information and Communication Technologies (ICT). This has been credited to the various projects that have been initiated in the computer and internet. In addition, Kiswahili is among the languages that have been accepted for use in ICT.

This paper discusses Kiswahili usage in ICT in two New Partnerships for Africa's Development (NEPAD) sponsored schools in Kenya that use Microsoft software: Chavakali and Maranda Boy's secondary schools. This study is guided by a model developed from a combination of two theories: Use and Gratification theory and Symbolic Interaction theory. Data from the two schools are analyzed using qualitative and quantitative analysis.
\end{abstract}

An attempt is also made to demonstrate that Kiswahili usage in ICT in school is influenced by the language used in teaching and learning in school. The data for this work came from 120 students selected through simple random sampling and 8 teachers of Kiswahili from the two schools selected through saturated sampling technique.

From the analysis of the responses from the study it is evident that a small percentage of teachers and students use Kiswahili in ICT in school. However, the study was able to come up with various uses of Kiswahili in ICT such as accessing the internet, learning and communicating with friends through email.

Keywords: ICT, NEPAD, Kiswahili, Kenya, Chavakali Boys Secondary school, Maranda Boys Secondary School.

\section{Background}

The word Information and Communication Technology (ICT) broadly refer to all forms of technology used to create, store, process and use information in its various forms (data, voice, image, multi media presentations and other forms including those not yet conceived) and which enable, facilitate and support communication. More specifically, ICT refer to the convergence of micro-electronics, computers and telecommunications which make it possible for data, including text, video and video signals, to be transmitted 
anywhere in the world where digital signals can be received. They include networks such as fixed, wireless and satellite telecommunications, broadcasting networks and applications such us the internet, database management systems and multi- medium tools (Howell and lundall, 2000). While ICT clearly encompass a wide range of technologies, for the purpose of this article ICT refer specifically to the use of computers, e- mail and internet use as these are the commonly used ICT in school.

The importance of ICT in education has been realized by many African countries and that is why various projects have been initiated in schools so as to integrate it into educations for example the NEPAD e-schools initiative projects through the NEPAD e- school Demo whose purpose is intended to accrue a body of knowledge, based on real- life experiences of the implementation of ICT in schools across the African continent that will serve to inform the roll-out of the broader NEPAD e- schools initiative. Cumulatively, these considerations give us the foundation for the discussions on the Kiswahili usage in ICT in schools. Kiswahili is one the languages that have been accepted for use in the various ICT. Therefore the potentials of Kiswahili usage in ICT would enhance global understanding.

\section{NEPAD}

The new partnership for Africa's development (NEPAD) is a vision and strategic frame work for Africa's renewal. The NEPAD strategic framework document arises from a mandate given to the five initiating Heads of State (Algeria, Egypt, Nigeria, Senegal and South Africa) by the Organization of African Unity (OAU) to develop an integrated 
socio-economic development framework for Africa. The $37^{\text {th }}$ summit of the AU in July 2001 formally adopted the strategic framework document. NEPAD is designed to address the current challenges facing the African continent. Issues such as the escalating poverty levels, underdevelopment and the continued marginalization of Africa needed a new radical intervention, spearheaded by African leaders, to develop a new vision that would guarantee Africa's renewal. The NEPAD e-Africa Commission is the NEPAD task team responsible for developing the NEPAD ICT program and implementing its projects (NEPAD e-Africa Commission, 2003).

\section{NEPAD e-schools in Kenya}

NEPAD e-School Demo project intends to impart ICT skills to young persons in primary and secondary schools and to use ICT to improve the provision of education in schools. Given that e-schools Demo project is to serve as a mechanism for learning in order to develop models for the effective roll-out of the NEPAD e- schools initiative, it is imperative that the schools Demo provide a reasonable reflection of the spectrum of African school environments. The participating schools were therefore chosen according to the following criteria:

1. All schools should be at a secondary or equivalent level.

2. There should be a mixture of urban and rural schools.

3. If possible, one of the schools should be without access to electricity.

4. There should be a mixture of schools in terms of size.

5. All schools should have a room that could be utilized for setting up an ICT laboratory. 
6. There should be security.

7. The teachers in these schools should be willing to avail themselves for training courses.

In Kenya six schools have already been selected initially to serve as demo schools for the implementation of the e-school project. These schools are situated in the six provinces: Nyanza, Western, Eastern, Central, North Eastern and Rift Valley. These six schools are inclusive of two boys school: Chavakali and Maranda, two mixed schools: Menengai and Isiolo and two girls schools: Mumbi and Wajir. Among these six schools three schools use Microsoft software's i.e. Maranda, Chavakali and Wajir while three schools use Oracle software's i.e. Mumbi, Isiolo and Menengai. These schools already have access to computers, internet facilities, Digital Satellite Television- DSTV, Smart board and projectors. The teachers and students in these schools have already been trained on how to use computers.

\section{Literature Review}

\subsection{Importance of Kiswahili in ICT}

Language is a very important factor in using ICT. It is language that facilitates the interaction that occurs between people and ICT. Herbert (1992) argues that the availability of software and content in the languages most familiar with users is an essential element in their adoption and optimal use of computers and the internet. In addition, in a context where people speak several languages as often finds in Africa - the option is empowering. 
Osborn (2007) states that the accommodation of the most familiar languages is a consideration of primary importance in any efforts to use ICT for development. He also adds that it should be of no surprise, as education and communication in the first languages in general is easier for people than in languages they acquire later.

Osborn (2007) notes that ICT was introduced to Africa in English, French, and in some countries south of the Sahara, Portuguese and Spanish- the same language of European origin that were used in colonization of these regions which have served as official language since their independence. These languages are not understood by majority of the population in these countries.

Moshi (2005) states that allowing English or other foreign languages to assume an exclusive role in Africa's technological advancement would, undoubtedly encourage dependency while thwarting global innovations that could be influenced by the African cultures and experiences. It would also encourage the divisions that exist between rural and urban communities, pitting those who live in cities/ towns against those who live in rural areas. In Kenya the majority of the population living in the rural areas does not understand English nor does it speak it well. Therefore Kiswahili offers an opportunity as an alternative language which can be used.

Mackey (1985) states that one problem with reliance on these languages is that a large majority of people on the continent either do not speak these languages or do not speak 
them well. Therefore in a country like Kenya where the majority speaks Kiswahili, it is important to give Kiswahili speakers the opportunity to use it in various ICT.

\subsection{The state of Kiswahili use in ICT}

There are various efforts that have been made to enable the use of Kiswahili in ICT. For example the growing use of Kiswahili in world media such as the voice of America Radio Deutsche Welle, BBC radio and Television, Asia radio and TV programs that comes to many homes in East Africa. In addition, Microsoft Windows and Microsoft Office in Kiswahili have been developed by Microsoft Company to enable the large population that speaks Kiswahili to use the computers (Microsoft Corporation, 2004).

The growing interest to expose Kiswahili to technology is also demonstrated in the move by Vodacom and Celtel (now Zain) companies to regularly place advertisements in newspapers in both English and Kiswahili to advertise their services. Currently, a spellchecker is in progress (spearheaded by Professor Arvi Hurskainen, University of Helsinki) to help editors who choose to write in Kiswahili (Ligere, 2005).

In addition, there is the Kamusi project which was initiated in 1994 which has produced Kiswahili-English and English-Kiswahili dictionaries which are used in the internet (Kamusi Project, Yale University).

Available is also the Swahili Language Management (SALAMA) which is important in developing the use of Kiswahili in computer (Sewangi, 2001). From these literatures it is 
therefore evident that there is credible effort that has been made to enable the use of Kiswahili in ICT. We therefore turn to the theoretical framework.

\section{Theoretical Framework}

In light of the foregrounding facts, Katz (1959) Use and Gratification theory and Mead (1934) Symbolic Interaction theory have been adopted as the theoretical construct of this study. The strength of these theories resides in their ability to provide a model to account for the interaction that occurs between ICT users and various ICT through language. Use and Gratification approach puts the function of linking need gratification and media choice clearly on the side of audience members. It suggests that people's needs influence what media they would choose, how they use certain media and what gratification the media gives them.

On the other hand, Mead (1934) claims that language is the most recognized symbol used by human beings. Mead agrees that interaction is used in every context of communication with an aim of involving people in societal activities.

A model is therefore developed from the two theories to be used in the study to show the interaction between users of ICT and ICT that is facilitated through language. 


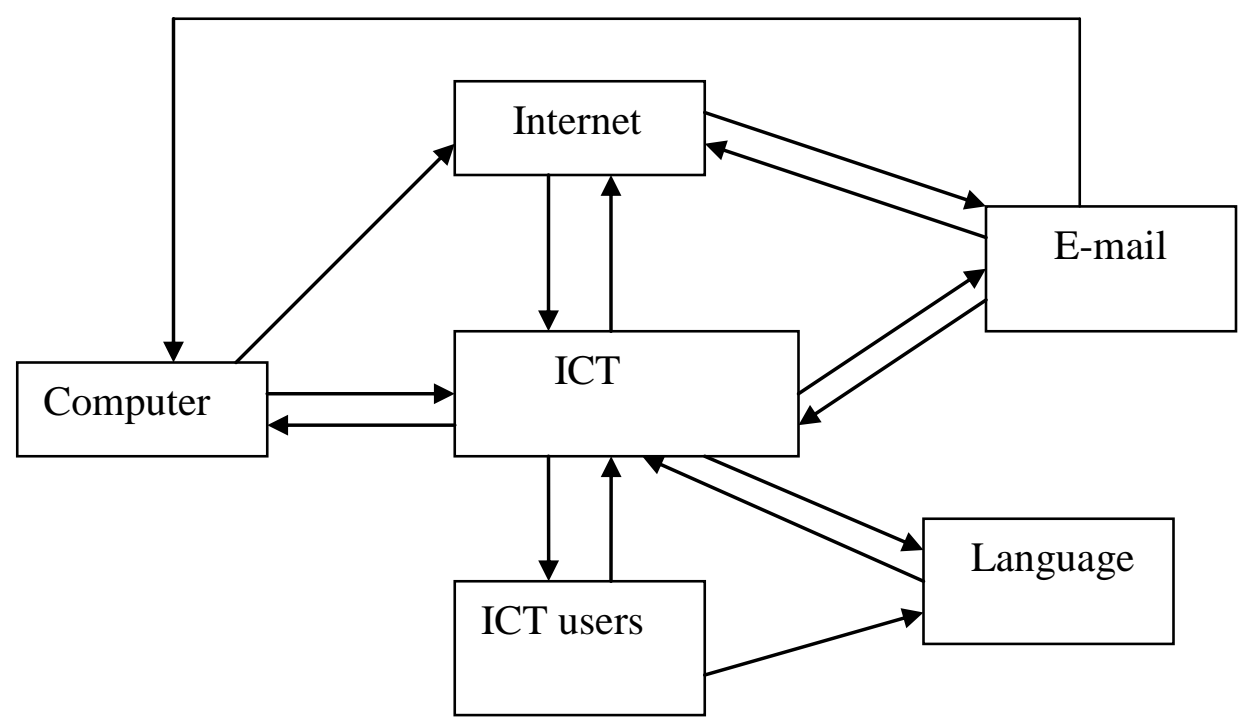

The model above shows the interaction that occurs between users, language and ICT. By looking at the various interactions, the study is able to derive various Kiswahili usages in the ICT.

\section{Methodology}

The data from the two schools were analyzed by using descriptions and tables with percentages. Sampling procedures were used in selecting the students and teachers who participated in the study. Simple random sampling was used to select 60 students in form three from each school because they all learn Kiswahili as a compulsory subject and they have also mastered how to use ICT. A total number of 4 teachers of Kiswahili from each school were selected by using saturated sampling because all the teachers who teach Kiswahili in form three were included. 


\section{Descriptions and Discussions}

In Kenya, apart from Kiswahili being a national language it is taught in schools as one of the compulsory subjects in both primary and secondary schools. It is believed that Kiswahili usage in ICT will have a direct impact on the development of Kiswahili in education through ICT. Since Kiswahili usage in ICT was the main concern of this study, discussions are therefore made on Kiswahili usage in ICT i.e. computer, internet and email.

Data on whether Kiswahili is used in ICT by students and teachers in school is important because it is difficult to explain Kiswahili usage in various ICT without explaining if Kiswahili is used in ICT by students and teachers. According to Symbolic Interaction theory by Mead (1934) people interact by using the language they understand. Therefore this study adopted this basis to determine whether teachers and students used Kiswahili in their interaction with ICT.

Table 1: Language preferred by students for computer use

\begin{tabular}{lcccc}
\hline & \multicolumn{2}{c}{ Chavakali } & \multicolumn{2}{c}{ Maranda } \\
\hline Language & No. of students & $\%$ & No. of students & $\%$ \\
\hline English & 53 & 88.33 & 52 & 86.67 \\
Kiswahili & 1 & 1.67 & - & - \\
English/ Kiswahili & 6 & 10.00 & 8 & 13.33 \\
\hline Total & 60 & 100 & 60 & 100 \\
\hline
\end{tabular}


Table 1 shows that only 1 student prefers to use Kiswahili in the Computer in Chavakali Boys secondary school. Majority of the students prefer to use English in the computers. One of the reasons given was that English is the language of instruction in all subjects except Kiswahili. Both English and Kiswahili languages are used in the computers as these are the languages that are used for communication in school. Despite the fact that only 1 student used Kiswahili in the computer, those students who used Kiswahili in the computers were able state how they use it in the computers.

Table 2: Ways in which students use Kiswahili in the computer

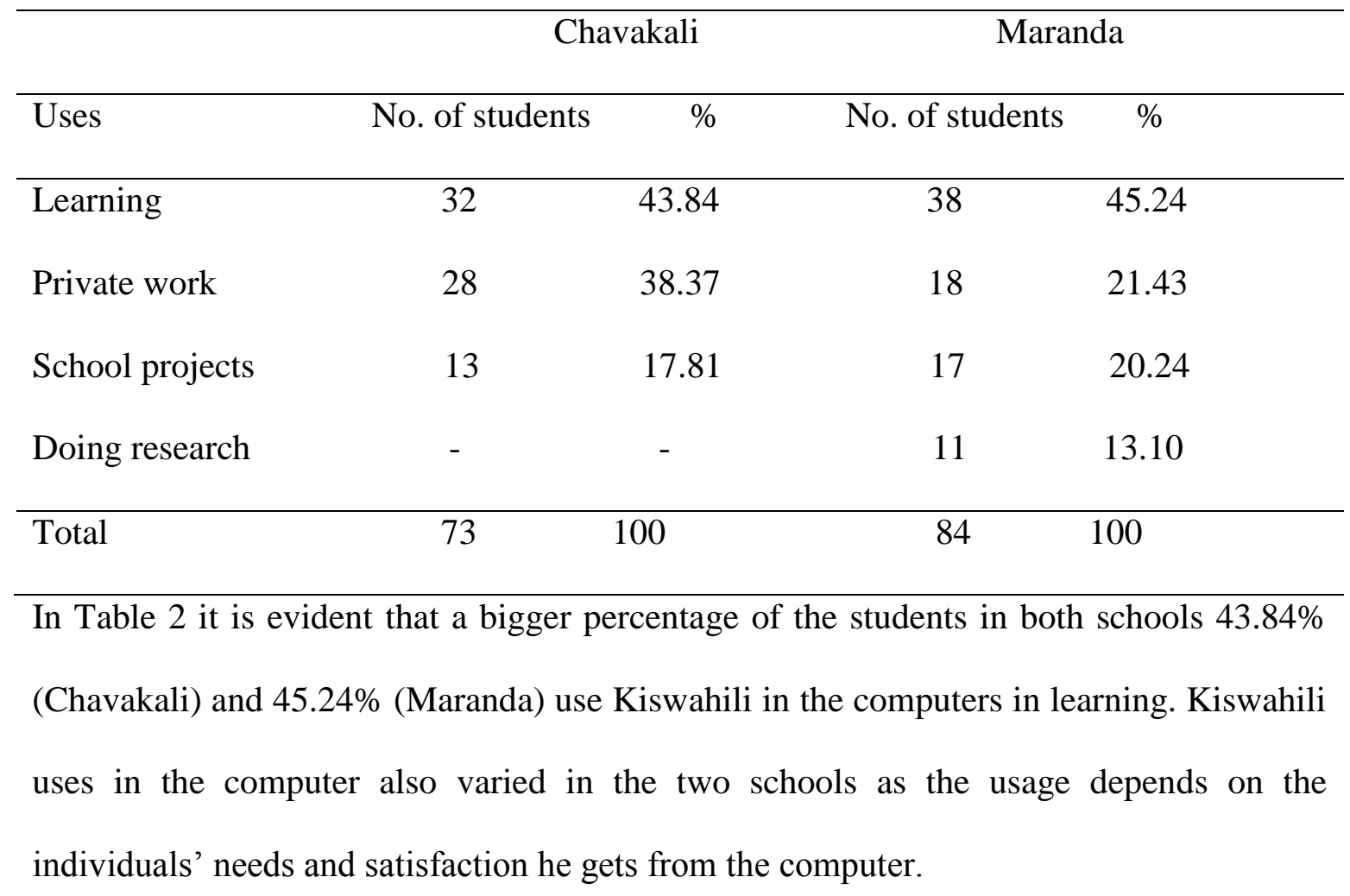

Table 3 Language used by teachers in the computer 


\begin{tabular}{lcccc}
\hline Language & No. of teachers & $\%$ & No. of teachers & $\%$ \\
\hline English & 3 & 75 & 3 & 75 \\
Kiswahili & - & - & - & - \\
English/Kiswahili & 1 & 25 & 1 & 25 \\
\hline Total & 4 & 100 & 4 & 100 \\
\hline
\end{tabular}

It is evident from Table 3 that only 1 teacher out of the 4 from each school prefers to use both English and Kiswahili while using the computers. One of the reasons given for using English and Kiswahili is that these are the languages they use in communication. Despite the fact that majority of the teachers use English, those who used Kiswahili were able to state how they use it in the computer.

Table 4: Ways in which teachers use Kiswahili in the computer

\begin{tabular}{lcccc}
\hline & \multicolumn{2}{c}{ Chavakali } & \multicolumn{2}{c}{ Maranda } \\
\hline Uses & No. of teachers & $\%$ & No. of teachers & $\%$ \\
\hline Preparation & 2 & 33.33 & 2 & 25.00 \\
School projects & 1 & 16.66 & 1 & 12.50 \\
Private work & 3 & 50 & 4 & 50.00 \\
Researching on Kiswahili & - & - & 1 & 12.50 \\
\hline Total & 6 & 100 & 8 & 100 \\
\hline
\end{tabular}


From the table it is evident that majority of the teachers from both schools use Kiswahili in the computer for their private work. There was variation in the usage as this depends on individual teacher.

Table 5: Language preferred by the students for use in the internet

\begin{tabular}{lcccc}
\hline & \multicolumn{2}{c}{ Chavakali } & Maranda \\
\hline Language & No. of teachers & $\%$ & No. of teachers & $\%$ \\
\hline English & 52 & 86.67 & 54 & 90.00 \\
Kiswahili & 1 & 1.67 & 1 & 1.67 \\
English/ Kiswahili & 7 & 11.67 & 5 & 8.33 \\
\hline Total & 60 & 100 & 60 & 100 \\
\hline
\end{tabular}

Table 7 shows that majority of the students prefer using English in the internet. Only 1 out of 60 students from both schools prefer using Kiswahili in the internet. Both English and Kiswahili are used in the internet by some students as these are languages mostly used in communication. Although only a small number of students use Kiswahili in the internet, those who used it were able mention how they use it.

Table 6: Ways in which students use Kiswahili in the internet

\begin{tabular}{lcccc}
\hline \multicolumn{2}{c}{ Chavakali } & \multicolumn{3}{l}{ Maranda } \\
\hline Uses & No. of students & $\%$ & No. of students & $\%$ \\
\hline Looking for information & 28 & 43.75 & 29 & 54.72 \\
Interacting with other students & 33 & 51.56 & 23 & 43.40 \\
Learning Kiswahili & 3 & 4.69 & 1 & 1.89 \\
\hline Total & 64 & 100 & 53 & 100 \\
\hline
\end{tabular}


It is evident from table 6 that majority of the students use Kiswahili while interacting with other students through the internet and also while looking for information. A small number of students from both schools use Kiswahili in the internet to learn Kiswahili.

Table 7: Language preferred for use by teachers in the internet

\begin{tabular}{lcccc}
\hline & \multicolumn{2}{c}{ Chavakali } & Maranda \\
\hline Language & No. of teachers & $\%$ & 2 & No. of teachers \\
\hline English & 3 & 75 & - & - \\
Kiswahili & - & - & 2 & 50 \\
English/ Kiswahili & 1 & 25 & 4 & 100 \\
\hline Total & 4 & 100 & & \\
\hline
\end{tabular}

Table 7 shows that majority of the teachers in Chavakali Boys secondary school prefer using English when using the internet. While it is evident that an equal number of teachers in Maranda Boys secondary school use English and English/Kiswahili in the internet, those teachers who used Kiswahili in the internet were able to mention how they used Kiswahili.

Table 8: Ways in which teachers use Kiswahili in the internet

\begin{tabular}{lcccc}
\hline & \multicolumn{2}{c}{ Chavakali } & \multicolumn{2}{c}{ Maranda } \\
\hline Uses & No. of teachers & $\%$ & No. of teachers & $\%$ \\
\hline Looking for information & 2 & 50 & 3 & 42.86 \\
Communication & - & - & 2 & 28.57 \\
Preparations & 2 & 50 & 2 & 28.57 \\
\hline
\end{tabular}


It is evident from table 8 that Kiswahili is used in the internet by teachers while looking for information, in communication and preparation. There were variations in the uses as internet can be used to carry out various activities depending on the individuals needs.

Table 9: Language preferred by students for use in e-mailing

\begin{tabular}{lcccc}
\hline & \multicolumn{2}{c}{ Chavakali } & \multicolumn{3}{c}{ Maranda } \\
\hline Language & No. of students & $\%$ & No. of students & $\%$ \\
\hline English & 39 & 65.00 & 7600 \\
Kiswahili & 11 & 18.33 & 14 & 11.67 \\
English/ Kiswahili & 10 & 16.67 & 3 & 5.00 \\
Other languages & - & - & 60 & 100 \\
\hline Total & 60 & 100 & & \\
\hline
\end{tabular}

Table 9 shows that a small percentage of students in both schools prefer to use Kiswahili in e-mailing. A comparison of the number of students using Kiswahili in e-mailing, computer and internet reveals that more students use Kiswahili in e-mailing than the latter two. This is an indication that language preferred for use in computer, internet or emailing depends on the context of use. Other languages which included mother tongue were also used in this context. An indication that e-mailing offers a social context where people can interact using any language known to them. 
Table 10: Ways in which students use Kiswahili in e-mailing

\begin{tabular}{|c|c|c|}
\hline Chavakali & & Maranda \\
\hline No. of students & $\%$ & No. of students $\%$ \\
\hline Communicating with friends \& families & 63.77 & 65.63 \\
\hline Exchanging information with other students 25 & 36.23 & 34.38 \\
\hline Total & 100 & 100 \\
\hline
\end{tabular}

Table 10 shows that majority of the students use Kiswahili in e-mailing. This is due to the fact that students prefer using Kiswahili while communicating with their friends and family members. Therefore e-mailing offers them a context where they can interact freely with friends and family members by using language that suits them best.

Table 11: Language preferred by teachers for use in e-mailing

\begin{tabular}{lcccc}
\hline & \multicolumn{2}{c}{ Chavakali } & \multicolumn{2}{c}{ Maranda } \\
\hline Languages & No. of teachers & $\%$ & No. of teachers & $\%$ \\
\hline English & 3 & 75 & 3 & 75 \\
Kiswahili & - & - & - & - \\
English/ Kiswahili & 1 & 25 & 1 & 25 \\
\hline Total & 4 & 100 & 4 & 100 \\
\hline
\end{tabular}

Table 11 shows that 3 teachers out of 4 in both schools prefer to use English in e-mailing. Only one out of 4 teachers from both schools prefers to use English/ Kiswahili in emailing. Both languages are used due to the fact that these languages are mostly used in communication. Despite the fact that only 1 teacher out 4 from each school prefer to use 
English/Kiswahili, those who used Kiswahili were able to mention how they use it in emailing.

Table 12: Ways in which teachers use Kiswahili in e-mailing

\begin{tabular}{lcccc}
\hline & Chavakali & \multicolumn{3}{l}{ Maranda } \\
\hline Uses & No. of teachers & $\%$ & No. of teachers & $\%$ \\
\hline Communicating with family members & - & - & 4 & 100 \\
They don't use & 4 & 100 & - & - \\
\hline Total & 4 & 100 & 4 & 100 \\
\hline
\end{tabular}

Table 12 shows that Kiswahili is only used in e-mailing by teachers in Maranda Boys School when communicating with family members. In Chavakali Boys school none of the teachers use Kiswahili in e-mailing. This is because majority of the teachers prefer to use English.

\section{Conclusion}

In this article, we have argued that Kiswahili usage in ICT in school is influenced by the language that is used in teaching and learning in school. Both English and Kiswahili languages are used by students and teachers in ICT because they are languages that are used in communication in school. Also noted is that a small percentage of teachers and students use Kiswahili in ICT because English is the language of instruction in all subjects except Kiswahili. 
Katz, Blumler \& Gurevitch (1974) points out that the use of ICT depends on individuals' needs and gratification they get from ICT. Data from the students and teachers on uses of Kiswahili in ICT shows variations proving that the uses of Kiswahili in ICT depend on individual's needs and the gratification they get. Various uses of Kiswahili in ICT have also been discussed such as accessing the internet for information, learning and communicating with friends and family members through e-mail.

\section{References}

Herbert, R. K. (1992). "Language in a divided Society” in R.K Herbert, ed. Language and Society in Africa: The theory and Practice of Sociolinguistics. Witwatersrand: Witwatersrand University Press. Pp. 1-9.

Howell, C. \& Lundall, P. (2000). Computers in schools: A National Survey of Information Communication and Technology in South African Schools. Education Policy Unit: University of the Western Cape: Cape Town.

http://www.school.za/schoolsurveys/surveys-index.htm accessed on 20th July 2006.

Jagero, J. A. (2009). Matumizi ya Kiswahili katika TEKNOHAMA katika shule za sekondari za Chavakali na Maranda nchini Kenya: Unpublished masters thesis, Maseno University.

Katz, E. (1959). Mass communication research and the study of culture. Journal of Studies in public Communication, 2, 1-6. 
Ligere, K. (2005). Uwezeshaji wa Kiswahili Siku Hizi, Mafanikio na Matatizo. A paper read at the 2005 Annual Conference on African Linguistics, Savannah, Georgia.

Mackey, W. F. (1989). "Status of Languages in Multinational Societies.” As quoted in Robinson (1996) Languages Use in Rural Development: An African Perspective. Berlin: Mouton De Gruyter.

Mead, G. H. (1934). Mind Self and Society. Chicago: University of Chicago.

Microsoft Corporation. (2004). "Microsoft Enables Millions More to Experience Personal Computing Through Local Languages Program.” Press-Pass-Information for Journalists, March 16, 2004.http://www.microsoft.com/presspass/press/2004/mar04/0316LLPPR.asp

Moshi, L. (2006). The Globalized World Languages: The case of Kiswahili. In Selected Proceedings of the $36^{\text {th }}$ Annual Conference on African Linguistics, Ed. Arasanyin O.F. \& Pemberton, M. A. Pemberton, 166-175. Somerville, MA: Cascadilla priceeding project.

NEPAD e-Africa Commission. (2003). The NEPAD e-Initiative: Ensuring that the young participate actively in the Global Information Society and Knowledge Economy.

Retrieved on October 22, 2007 at http//www.nepadafricacommission.org 
Osborn, D. Z. (2007). A Survey of Localization in African languages, its prospects: A Background Document. Panfrican: California.

Sewangi, S. (2001). Computer-Assisted Extraction of Terms in Specific Domains: The case of Kiswahili. Ph.D. Thesis. Publication of Institute for Asian and African studies, University of Helsinki. 